\title{
Efeito da Estrutura de uma Pastagem Hibernal sobre o Comportamento de Pastejo de Novilhos de Corte
}

\author{
Naíme de Barcellos Trevisan', Fernando Luiz Ferreira de Quadros², Alexandre Coradini \\ Fontoura da Silva ${ }^{3}$, Duílio Guerra Bandinelli ${ }^{4}$, Carlos Eduardo Nogueira Martins ${ }^{5}$
}

\begin{abstract}
RESUMO - Avaliou-se o efeito de diferentes estruturas da pastagem, provocadas pela imposição de dois níveis (tratamentos) de biomassa de lâminas foliares verdes (MSFV) - 350 e $600 \mathrm{~kg} / \mathrm{ha}$ de matéria seca (MS) - sobre o comportamento de pastejo dos animais em pastagem consorciada de aveia preta (Avena strigosa Schreb.) e azevém anual (Lolium multiflorum Lam.). As atividades dos animais foram registradas nos meses de agosto e setembro, em períodos de 24 horas consecutivas, por observadores treinados. Nas horas do dia em que o pastejo é mais intenso, foram avaliados o tempo de permanência nas estações de pastejo, a distância entre as estações alimentares e a freqüência com que os animais permaneciam em um sítio de pastejo. Houve preferência marcante por sítios localizados nos quadrantes de cada potreiro mais próximos dos bebedouros/fontes de água. O tempo de permanência dos animais nas estações de pastejo e a distância entre elas foram similares entre os dois tratamentos. O tratamento de $350 \mathrm{~kg} / \mathrm{ha}$ de MS de lâminas foliares verdes não se constituiu como um nível de biomassa que afeta o tempo de permanência nas estações de pastejo.
\end{abstract}

Palavras-chave: estações alimentares, sítio de pastejo, lâminas foliares

\section{Effect of Structure of a Cool Season Pasture on Grazing Behaviour of Beef Steers}

ABSTRACT - The effect of different pasture structures imposed by treatments of green leaf lamina biomass (GLLB) levels of 350 and $600 \mathrm{~kg} / \mathrm{ha}$ of dry matter (MS), over animals grazing behaviour was evaluated in this experiment. The pasture was oat (Avena strigosa Schreb.) and Italian ryegrass (Lolium multiflorum Lam.). Animals activities were recorded in August and September, in 24 consecutive hours, by trained observers. In the hours of the day in which grazing were more intense, grazing stations remaining time, distance between feed stations and the frequency of remaining in each grazing site were recorded. Preference for sites located nearest to drinking points were found, in each paddock. Distances between feeding stations and time of remaining in these were similar for both treatments. The treatment with $350 \mathrm{~kg} / \mathrm{ha}$ of DM of GLLB was not a biomass level that affect remaining time in grazing stations.

Key Words: feeding stations, grazing site, leaf lamina

\section{Introdução}

Graças à capacidade seletiva dos herbívoros, a forragem colhida é, muitas vezes, superior em qualidade à média representativa do total ofertado (DenardinSaldanha, 1989; Soares, 2001). Portanto, os mecanismos que regulam o processo de pastejo incluem situações de preferência por determinados sítios, os quais comportam um conjunto de estações alimentares ou agregados de manchas de pastejo em uma pastagem (Bailey et al., 1996; Carvalho et al., 2001).

Os fatores quali-quantitativos determinantes da preferência por estes sítios são a quantidade de nutrientes minerais disponíveis nas plantas escolhidas, sobretudo de nitrogênio e enxofre, o local topo- gráfico em que se situam (Arnold \& Dudzinski, 1978; Soares, 2001), o fácil acesso às folhas, de acordo com sua distribuição espacial na comunidade vegetal, e a elevada relação folha:colmo (Carvalho et al., 2001; Trevisan et al., 2003).

O processo de seleção de forragem pode definir variações estruturais em diferentes dimensões no plano da vegetação, resultantes da relação entre $o$ animal e a planta, que é determinante dos índices de produtividade obtidos com o manejo proposto para as espécies forrageiras disponíveis. Para adequada compreensão destes índices, é necessária a descrição tanto da estrutura da pastagem ao longo de seu ciclo, quanto dos componentes do comportamento ingestivo, destacando-se a distância entre os locais pastejados e

\footnotetext{
${ }^{1}$ Aluna de Graduação em Zootecnia - UFSM. Bolsista PIBIC-CNPq. Av. Medianeira, 1286/801, CEP: 9706-002, Centro, Santa Maria, RS. Endereço eletrônico: naime.trevisan@zipmail.com.br. Autora para correspondências.

2 Eng. Agr., Dr. Prof. Adjunto do Departamento de Zootecnia - UFSM (fquadros@ccr.ufsm.br).

${ }^{3}$ Aluno de Graduação em Zootecnia - UFSM. Bolsista FIPE (salso@vant.com.br).

${ }^{4}$ Eng. Agr., Aluno de Pós-Graduação em Zootecnia - UFSM. Bolsista CAPES (bandlli@zipmail.com.br)

${ }^{5}$ Aluno de Graduação em Zootecnia - UFSM. Bolsista BIC-FAPERGS (cenmartins@ieg.com.br).
} 
o tempo que os animais permanecem nas estações de pastejo, como resultado das interações entre os ruminantes e as estruturas horizontal (Laca \& Lemaire, 2000) e vertical da pastagem (Cangiano et al., 2002; Trevisan et al., 2003).

A quantidade de matéria seca e, principalmente, a disponibilidade de folhas verdes acessíveis nos horizontes superficiais da pastagem afetam o tempo de permanência dos ruminantes na busca e colheita do alimento. A facilidade de apreensão da forragem é um dos fatores determinantes de aumentos ou reduções no tempo de pastejo e, conseqüientemente, de alterações nos tempos de ruminação, ócio, atividades sociais, entre outros, posto que as atividades do comportamento ingestivo são excludentes (Carvalho et al., 2001; Silva et al., 2003b).

De modo geral, qualquer pastagem é heterogênea, porque, ainda que formada por apenas um tipo de espécie, existem variações de massa de forragem, aliadas a áreas de maior ou menor fertilizade dentro dos proteiros. Esta heterogeneidade afeta a quantidade e qualidade da forragem ingerida, contribuindo para a obtenção de diferentes níveis de produção animal para um mesmo valor de oferta de forragem (Carvalho, 1997).

Este trabalho foi conduzido com o objetivo de verificar o efeito da estrutura da pastagem, provocada por diferentes biomassas de lâmina foliar verde, sobre o comportamento de busca e escolha de forragem por novilhos de corte em pastagem consorciada de aveia preta (Avena strigosa Schreb.) e azevém anual (Lolium multiflorum Lam.).

\section{Material e Métodos}

O experimento foi conduzido no Departamento de Zootecnia da Universidade Federal de Santa Maria (UFSM), no município de Santa Maria, que está situado nas coordenadas $29^{\circ} 43^{\prime}$ de latitude sul e $53^{\circ}$ 42' de longitude oeste, com $95 \mathrm{~m}$ de altitude, na Depressão Central do Estado do Rio Grande do Sul. O clima, segundo a classificação de Köppen (Moreno, 1961), é Cfa - subtropical úmido.

A área experimental constituiu-se de 5,4 hectares, subdivididos em seis potreiros de 0,7 hectare e um potreiro de 1,2 hectare para a permanência dos animais reguladores. $\mathrm{O}$ estabelecimento da pastagem ocorreu no dia $11 / 05$, por meio de semeadura direta, utilizando $84 \mathrm{~kg} / \mathrm{ha}$ de sementes de aveia preta em linhas e $40 \mathrm{~kg} / \mathrm{ha}$ de sementes de azevém a lanço. Em razão da baixa germinação das sementes de azevém, realizou-se nova semeadura de azevém no dia $25 / 05$, com $25 \mathrm{~kg} / \mathrm{ha}$ de sementes.

A adubação de base ocorreu por ocasião da semeadura da aveia, com auxílio de uma semeadoraadubadora, utilizando-se $200 \mathrm{~kg} / \mathrm{h}$ a da fórmula 05-20-20 (NPK). Em cobertura, foram aplicados $100 \mathrm{~kg} / \mathrm{ha}$ de $\mathrm{N}$ na forma de uréia, divididos em duas aplicações, nos dias 20/06 e 12/08.

O método de pastejo foi o contínuo com taxa de lotação variável, conforme metodologia proposta por Mott \& Lucas (1952). Foram utilizados 33 novilhos inteiros da raça Charolês e suas cruzas com Nelore, com nove meses de idade e peso médio inicial de $175 \mathrm{~kg}$, sendo empregados três animais testes e um número variável de reguladores por potreiro conforme a biomassa de lâminas foliares pretendida. Para isto, a cada 14 dias foram realizadas estimativas de massa de forragem e de biomassa de lâminas foliares verdes pelo método de estimativa visual direta com dupla amostragem (t'Manetje, 2000). A partir das amostras cortadas, foram determinados os componentes lâmina de folha, colmo + bainha e material senescente da forragem disponível, por meio de separação manual, pesagem e secagem até peso constante (Silva et al., 2003a).

Os tratamentos propostos consistiram de dois níveis de biomassa de lâmina foliar verde (MSFV), correspondentes a 350 (baixa biomassa) e $600 \mathrm{~kg} / \mathrm{ha}$ (alta biomassa) de MS em pastagem cultivada de aveia preta e azevém anual. Cada potreiro foi considerado uma repetição de um tratamento.

Para análise do comportamento ingestivo, foram utilizados 18 novilhos focais (três em cada potreiro), usados como testes para avaliações da produção animal.

O comportamento ingestivo foi avaliado por observadores treinados, um para cada dois potreiros, durante um período de seis horas consecutivas, durante 24 horas consecutivas, em duas ocasiões durante o período de utilização da pastagem (16-17/08 e 24-25/09/ 2002). As avaliações foram realizadas simultaneamente às observações das atividades de tempos de pastejo, ruminação e ócio (minutos/dia) e taxa de bocados (número de bocados/minuto). Estas observações foram feitas pela escolha aleatória de um dos animais em intervalos de 10 minutos para cada período de seis horas, quando os três animais testes de cada 
potreiro tinham suas atividades registradas e a taxa de bocados medida.

As temperaturas mínimas verificadas nos dias $16-17 / 08$ e $24-25 / 09$ foram de 12,4 e $7,2^{\circ} \mathrm{C}$, respectivamente, e as temperaturas máximas de 26,8 e $24,4^{\circ} \mathrm{C}$. As médias de insolação corresponderam a quatro horas e 26 minutos e 10 horas e 12 minutos, para o primeiro e segundo períodos, respectivamente.

Nos períodos do dia de maior freqüência de pastejo, pela manhã, de $7 \mathrm{~h}$ às $9 \mathrm{~h}$, e pela tarde, de $16 \mathrm{~h}$ às $18 \mathrm{~h}$ (Arnold \& Dudzinski, 1978; Quadros et al., 2003), foram anotados, em instantâneos com intervalos de 10 minutos, o tempo de permanência do animal na estação alimentar, a distância média entre as estações e o tempo de permanência do animal dentro de um sítio de pastejo.

Uma estação alimentar é definida como o espaço correspondente ao pastejo sem movimentos das patas dianteiras (Laca et al., 1992). A distância média entre estas estações foi calculada como o número de passos dados pelo animal (transformados em metros lineares) entre uma estação e outra. Para medir o tempo de permanência do animal dentro de um "agregado de manchas de pastejo em uma área contígua (sítio de pastejo)" (Bailey, 1996; Carvalho et al., 2001), foram demarcados quatro quadrantes em cada potreiro.

Os quadrantes 'um' e 'quatro', localizados ao norte do potreiro, corresponderam aos locais de baixada com bebedouros ou outras fontes de água. Os quadrantes 'dois' e 'três', ao sul do potreiro, representaram as áreas de topo de coxilha e encosta e corresponderam ao local "reservado" ao descanso dos animais durante a noite.

Para caracterizar a estrutura vertical, foi registrada a altura da pastagem em 20 pontos escolhidos ao acaso. Destes, cinco pontos foram selecionados, nos quais a forragem foi cortada em camadas verticais de cinco a $15 \mathrm{~cm}$ utilizando-se para isto quadros de $0,40 \mathrm{~m}^{2}$. As amostras foram separadas manualmente em folhas verdes, pseudocaule e material morto, obtendose a quantidade absoluta de cada componente, em cada intervalo de cinco centímetros de altura da pastagem. Estes intervalos corresponderam aos estratos da pastagem. Conforme Cangiano et al. (2002), os bovinos concentram o pastejo nos horizontes superficiais da pastagem, caracterizados pelos estratos de mais fácil acesso, ou seja, os estratos superiores. No tratamento de $350 \mathrm{~kg} / \mathrm{ha}$ de MSFV, estes estratos correspondem às médias da massa de forragem nas alturas de cinco e $10 \mathrm{~cm}$ e, no tratamento de alta biomassa, nas alturas de 10 e $15 \mathrm{~cm}$.

A estrutura horizontal da pastagem foi avaliada visualmente, separando-se áreas de maior e menor alturas (conforme as medidas supracitadas), caracterizadas como manchas de sub e superpastejo, em cada um dos quadrantes do potreiro.

O delineamento experimental adotado foi o inteiramente casualisado com número variável de repetições, sendo duas para o tratamento de 350 e quatro para o tratamento de $600 \mathrm{~kg} / \mathrm{ha} \mathrm{MSFV}$, uma vez que duas repetições do tratamento de $600 \mathrm{~kg} / \mathrm{ha}$ MSFV deveriam ser repetições de um tratamento com $900 \mathrm{~kg} / \mathrm{ha}$ MSFV, Em virtude da baixa fertilidade do solo da área experimental, não foi possível atingir este nível mais alto, exigindo que estes potreiros fossem manejados como no tratamento de $600 \mathrm{~kg} / \mathrm{ha}$ de MSFV.

As distâncias entre as estações alimentares foram analisadas estatisticamente entre os tratamentos, os turnos (manhã e tarde) nas hora correspondentes aos picos de pastejo e períodos (16-17/08 e 24-25/09). $\mathrm{O}$ mesmo critério foi empregado para analisar os tempos de permanência dos animais nas estações alimentares.

A frequiência de observações dos animais focais nos sítios de pastejo correspondeu à porcentagem de observações em determinado sítio em relação ao total de observações nos demais sítios. Esta variável foi analisada estatisticamente entre tratamentos e períodos.

Para a análise das variáveis estimadas, utilizou-se a rotina de testes de aleatorização do programa MULTIV (Pillar, 1997), empregando-se o seguinte modelo estatístico:

$$
\gamma \mathbf{i j}=\mu+\tau \mathbf{i}+\varepsilon \mathbf{i j}
$$

em que $\gamma$ ij é a observação do tratamento i na repetição $\mathrm{j} ; \mu$, a média geral; $\tau \mathrm{i}$, o efeito do tratamento i; e $\varepsilon \mathrm{ij}$, o erro associado à observação $\mathrm{j}$.

\section{Resultados e Discussão}

Os valores de distância média entre as estações alimentares, por período de avaliação e turno do dia, são apresentados na Tabela 1. Todas as atividades relacionadas ao comportamento ingestivo de ruminantes em pastejo estão distribuídas de maneira que o propósito de maximização do consumo de forragem pelo animal seja alcançado (Charnov, 1976; Stephen 
Tabela 1- Distância média (metros lineares) entre estações alimentares de pastejo por tratamento e por turno do dia nos dois períodos avaliados

Table 1 - Average distance (linear meters) between feeding stations by treatments and by daily turn in the two evaluation periods

\begin{tabular}{|c|c|c|c|}
\hline $\begin{array}{l}\text { Período } \\
\text { Period }\end{array}$ & $\begin{array}{c}350 \mathrm{~kg} / \\
\text { ha MSFV } \\
350 \mathrm{~kg} / \\
\text { ha DMLL }\end{array}$ & $\begin{array}{c}600 \mathrm{~kg} / \\
\text { ha MSFV } \\
600 \mathrm{~kg} / \\
\text { ha DMLL }\end{array}$ & $\begin{array}{l}\text { Média } \\
\text { Average }\end{array}$ \\
\hline \multicolumn{4}{|c|}{$\begin{array}{l}16 / 08 / 02 \\
08 / 16 / 02 \\
\end{array}$} \\
\hline $\begin{array}{l}\text { Manhã } \\
\text { Morning }\end{array}$ & 3,44 & 2,57 & $3,00 \mathrm{a}$ \\
\hline $\begin{array}{l}\text { Tarde } \\
\text { Afternoon }\end{array}$ & 7,01 & 4,43 & $5,72 b$ \\
\hline $\begin{array}{l}\text { Média } \\
\text { Average }\end{array}$ & 5,22 & 3,50 & $4,36 \mathrm{~A}$ \\
\hline \multicolumn{4}{|c|}{$\begin{array}{l}24 / 09 / 02 \\
09 / 24 / 02 \\
\end{array}$} \\
\hline $\begin{array}{l}\text { Manhã } \\
\text { Morning }\end{array}$ & 2,12 & 2,06 & $2,09 a$ \\
\hline $\begin{array}{l}\text { Tarde } \\
\text { Afternoon }\end{array}$ & 2,69 & 2,74 & $2,71 \mathrm{a}$ \\
\hline $\begin{array}{l}\text { Média } \\
\text { Average }\end{array}$ & 2,40 & 2,40 & $2,40 \mathrm{~B}$ \\
\hline
\end{tabular}

Médias seguidas de letras maiúsculas distintas diferem $(P \leq 0,06)$ entre si.

Médias seguidas de letras minúsculas distintas diferem $(P \leq 0,10)$ entre si.

Means followed by different capital letters differ ( $P \leq .06)$.

Means followed by different small letters $\operatorname{differ}(P \leq .10)$.

\& Krebs, 1986). Quanto maior a distância percorrida entre as estações alimentares, maior é a seletividade de forragem exercida pelos animais, embora situações de aumento nas distâncias percorridas possam ser relativas a baixas disponibilidades de forragem (Rouge et al. 1998).

Ocorreram diferenças significativas entre os turnos avaliados dentro do primeiro período, no qual as médias do turno matinal representaram praticamente $50 \%$ dos valores encontrados para as médias do turno da tarde, indicando pastejo mais intenso e com menor seletividade pela manhã. Este registro contradiz a literatura consultada, que indica o pastejo vespertino como o menos seletivo (Arnold \& Dudzinski, 1978). Não houve diferenças entre os turnos do dia para as distâncias entre estações de pastejo no segundo período.

Nas duas datas de avaliações do comportamento ingestivo, não foram verificadas diferenças entre as médias das distâncias percorridas na busca por estações alimentares em cada tratamento, o que indica que o nível de $350 \mathrm{~kg} / \mathrm{ha}$ de MSFV não se constitui uma quantidade de biomassa limitante ao encontro destas estações.

De acordo com Dumont et al. (1995), o nível de biomassa de folhas verdes de azevém perene que provocaria alterações no comportamento de escolha por estações alimentares, obrigando os animais a consumirem plantas em estádio reprodutivo, seria de $300 \mathrm{~kg} / \mathrm{ha} \mathrm{MSFV}$, representado por uma altura média de $9 \mathrm{~cm}$. Neste caso, o animal caminha mais (Rouge et al., 1998), aumenta o tempo diário total de pastejo (Prache et al., 1998) ou aprende sobre os locais de disponibilidade de alimento usando a memória espacial junto à visual (Edwards et al., 1997; Dumont et al., 1998). As duas últimas afirmações podem ser a explicação para não terem sido encontradas diferenças nas distâncias percorridas entre as estações alimentares quando se analisaram os tratamentos deste experimento, durante o primeiro período.

$\mathrm{Na}$ data da primeira avaliação do comportamento ingestivo, a altura média registrada da pastagem foi de 8 e 21,6 cm para os tratamentos de baixa e alta biomassa, respectivamente. O tempo diário de pastejo verificado nestas avaliações é de 618,33 e 531,67 minutos por dia, distintos significativamente (Pd"0,049) entre os tratamentos de 350 e $600 \mathrm{~kg} / \mathrm{ha}$ de MSFV, respectivamente, conforme descrito por Silva et al. (2003b).

Considerando as características da vegetação como influentes na escolha de estações alimentares, duas situações podem ser distinguidas: quando os animais podem pastejar em suas estações preferidas sem ter que "buscá-las" ou quando a procura por estações de pastejo modifica as preferências (Baumont et al., 2000). Os resultados encontrados neste trabalho indicam que houve menor procura por estações alimentares no mês de setembro, em comparação ao mês de agosto nos dois tratamentos. Conforme Black \& Kenney (1984), quando a procura é insignificante, os animais geralmente preferem estações nas quais podem comer rapidamente. Neste caso, a preferência do animal é decorrente da estrutura vertical da planta (Harvey \& Orr, 1996; Prache et al., 1996).

Na Tabela 2, é apresentado o tempo médio de permanência nas estações alimentares. A acessibilidade das folhas verdes nas alturas superficiais do pastejo, por meio de sua distribuição espacial, e a relação folha:colmo ao longo do ciclo de desenvolvimento da pastagem podem interferir no tempo que o animal ocupa para a apreensão da forragem em uma estação de pastejo. 
Tabela 2 - Tempo médio de permanência nas estações alimentares (segundos) por turno do dia nos dois períodos avaliados

Table 2 - Average staying time in feeding stations (seconds) by daily turn in the evaluations

\begin{tabular}{|c|c|c|c|}
\hline \multicolumn{4}{|c|}{$\begin{array}{c}\text { Tratamento } \\
\text { Treatment }\end{array}$} \\
\hline $\begin{array}{l}\text { Período } \\
\text { Period }\end{array}$ & $\begin{array}{c}350 \mathrm{~kg} / \\
\text { ha MSFV } \\
350 \mathrm{~kg} / \\
\text { ha DMLL }\end{array}$ & $\begin{array}{c}600 \mathrm{~kg} / \\
\text { ha MSFV } \\
600 \mathrm{~kg} / \\
\text { ha DMLL }\end{array}$ & $\begin{array}{l}\text { Média } \\
\text { Average }\end{array}$ \\
\hline \multicolumn{4}{|c|}{$\begin{array}{l}16 / 08 / 02 \\
08 / 16 / 02 \\
\end{array}$} \\
\hline $\begin{array}{l}\text { Manhã } \\
\text { Morning }\end{array}$ & 21,62 & 17,81 & $19,71 \mathrm{a}$ \\
\hline $\begin{array}{l}\text { Tarde } \\
\text { Afternoon }\end{array}$ & 14,29 & 18,73 & $16,51 \mathrm{a}$ \\
\hline $\begin{array}{l}\text { Média } \\
\text { Average }\end{array}$ & 17,95 & 18,27 & $18,11 \mathrm{a}$ \\
\hline \multicolumn{4}{|c|}{$\begin{array}{l}24 / 09 / 02 \\
09 / 24 / 02 \\
\end{array}$} \\
\hline $\begin{array}{l}\text { Manhã } \\
\text { Morning }\end{array}$ & 20,57 & 21,28 & $20,92 \mathrm{a}$ \\
\hline $\begin{array}{l}\text { Tarde } \\
\text { Afternoon }\end{array}$ & 18,62 & 16,06 & $17,34 a$ \\
\hline $\begin{array}{l}\text { Média } \\
\text { Average }\end{array}$ & 19,59 & 18,67 & $19,13 a$ \\
\hline
\end{tabular}

Médias seguidas de letras minúsculas distintas diferem $(P \leq 0,10)$ entre si.

Means followed by different small letters differ $(P \leq .10)$.
Segundo Silva et al. (2003a), a concentração de folhas neste experimento esteve próxima a $50 \%$ durante todo o período de utilização da pastagem, com pequenas variações, justificando a semelhança entre os tratamentos, turnos e períodos de avaliação para o tempo que os animais permaneciam nas estações alimentares.

Houve menor relação folha:colmo média para o tratamento de $600 \mathrm{~kg} / \mathrm{ha}$ MSFV (Tabela 3), em comparação ao tratamento de baixa biomassa, nos estratos superiores de pastejo, apesar da maior biomassa de lâminas foliares e de colmos no tratamento de alta MSFV.Estes dois fatores (relação folha:colmo e biomassa destes componentes) podem ter tido um efeito de complemento, ocasionando volume de bocados semelhantes, apenas em estratos de pastejo distintos.

Esta possibilidade é reforçada em função de o volume de bocados ser um dos itens que compõem o cálculo do consumo de forragem, que, por sua vez, influencia o desempenho animal. Silva et al. (2003a) relatam que não foram verificadas diferenças, na média, em ganho de peso médio diário dos animais nestes tratamentos.

Os valores de freqüência de pastejo por sítio são apresentados na Tabela 4. Considerando que estas

Tabela 3 - Densidade de lâminas foliares e colmos $\left(\mathrm{kg} / \mathrm{cm}^{2}\right.$ de MS) e relação folha:colmo nos estratos superiores da pastagem, de acordo com os tratamentos e datas de avaliações

Table 3 - Leaf blade and stem density $\left(\mathrm{kg} / \mathrm{cm}^{2}\right.$ of $\left.D M\right)$ and leaf $/$ stem rate in the upper grazing layers, according to the treatments and evaluation dates

\begin{tabular}{|c|c|c|c|c|c|c|}
\hline \multirow[b]{3}{*}{$\begin{array}{l}\text { Data } \\
\text { Date }\end{array}$} & \multicolumn{6}{|c|}{$\begin{array}{c}\text { Tratamento }\left(\mathrm{kg} / \mathrm{cm}^{2} \mathrm{MS}\right) \\
\text { Treatment }\left(\mathrm{kg} / \mathrm{cm}^{2} \mathrm{DM}\right)\end{array}$} \\
\hline & 350 & 600 & 350 & 600 & 350 & 600 \\
\hline & \multicolumn{2}{|c|}{$\begin{array}{l}\text { Lâmina foliar } \\
\text { Leaf blade }\end{array}$} & \multicolumn{2}{|c|}{$\begin{array}{c}\text { Colmo } \\
\text { Stem }\end{array}$} & \multicolumn{2}{|c|}{$\begin{array}{l}\text { Relação folha:colmo } \\
\text { Leaf/stem rate }\end{array}$} \\
\hline $\begin{array}{l}10 / 08 / 02 \\
08 / 10 / 02\end{array}$ & 11,33 & 50,60 & 14,73 & 41,80 & 0,77 & 1,21 \\
\hline $\begin{array}{l}13 / 09 / 02 \\
09 / 13 / 02\end{array}$ & 14,59 & 11,60 & 2,14 & 14,31 & 6,81 & 0,81 \\
\hline $\begin{array}{l}\text { Média } \\
\text { Average }\end{array}$ & $12,96 \mathrm{~B}$ & $31,10 \mathrm{~A}$ & $8,43 \mathrm{~B}$ & $28,05 \mathrm{~A}$ & $3,79 \mathrm{~A}$ & $1,01 \mathrm{~A}$ \\
\hline
\end{tabular}

Médias seguidas de letras distintas indicam diferenças $(P \leq 0,06)$.

Means followed by different letters differ $(P \leq .06)$. 
foram semelhantes entre os turnos, conforme descrito por Quadros et al. (2003), são apresentadas como médias diárias.

Pode ser observado que a escolha dos sítios de pastejo não foi influenciada pelos níveis de biomassa. O fato relatado por Silva et al. (2003a) de que a oferta de forragem média nos dois tratamentos não parece ter limitado o desempenho animal pode ter contribuído para a ausência de preferência por sítios que pudessem ser atribuídas à variável biomassa de lâminas foliares verdes. Apesar disso, a estrutura dos potreiros de alta biomassa era visivelmente mais heterogênea que nos de baixa biomassa, que poderia ser caracterizada por maior quantidade de estações de subpastejo, especialmente nas áreas de encosta ou topo de coxilha. Essa observação está de acordo com Arnold \& Dudzinski (1978), que verificaram que cerca de $56 \%$ da variação no uso de sítios de pastejo pode ser atribuída a fatores relacionados à topografia do potreiro.

Verifica-se também que, durante o mês de agosto, os picos de pastejo concentravam-se nos quadrantes 'dois' e 'três', que são afastados dos bebedouros. Esse comportamento foi invertido em setembro, quando os animais concentraram o pastejo nos locais mais próximos das fontes de água disponíveis, o que pode ser parcialmente explicado pelo elevado nível de insolação verificado nos dias 24-25/09, 10h e $12 \mathrm{~min}$, em comparação aos dias 16-17/08, 4h e 26 min. Esta

Tabela 4 - Freqüência de observações da permanência dos animais em cada sítio de pastejo, em \% relativa à permanência destes nos demais sítios

Table 4 - Frequency of observations of staying of animals in each grazing site in \% relative to staying time in the other sites

\begin{tabular}{|c|c|c|c|c|}
\hline \multicolumn{2}{|c|}{$\begin{array}{l}\text { Tratamento } \\
\text { (kg/ha MSFV) }\end{array}$} & \multicolumn{2}{|c|}{$\begin{array}{l}\text { Sítio pastejo } \\
\text { Grazing site }\end{array}$} & \\
\hline & \multicolumn{4}{|c|}{$\begin{array}{c}16 \mathrm{e} 17 / 08 / 02 \\
08 / 16 \text { and } 08 / 17 / 02\end{array}$} \\
\hline & 1 & 2 & 3 & 4 \\
\hline 350 & 5,77 & 41,52 & 34,04 & 18,67 \\
\hline 600 & 25,16 & 10,58 & 29,95 & 34,31 \\
\hline Média & 15,47 & 26,05 & 37,00 & 26,49 \\
\hline \multicolumn{5}{|l|}{ Average } \\
\hline & \multicolumn{4}{|c|}{$\begin{array}{c}24 \text { e } 25 / 09 / 02 \\
09 / 24 \text { and } 09 / 25 / 02\end{array}$} \\
\hline 350 & 24,41 & 11,31 & 24,40 & 39,88 \\
\hline 600 & 34,40 & 15,13 & 31,97 & 18,50 \\
\hline Média & 29,41 & 13,22 & 28,19 & 29,19 \\
\hline Average & & & & \\
\hline
\end{tabular}

preferência já foi registrada por outros autores, como Arnold \& Dudzinski (1978) e Bagshaw et al. (2000), que relataram que bovinos preferem locais mais planos próximos a cursos de água, mesmo que existam outros bebedouros disponíveis em situação de encosta.

\section{Conclusões}

A distância entre as estações alimentares não foi afetada pelos tratamentos impostos, mas foi influenciada pelo período em que foi avaliada.

O tempo de permanência do animal nas estações alimentares não foi afetado pela biomassa de MS foliar, pelo turno do dia ou pelo mês do ano avaliados.

A escolha de sítios de pastejo é mais dependente da posição topográfica e localização das aguadas que dos níveis de biomassa de lâminas foliares verdes.

\section{Literatura Citada}

ARNOLD, G.W; DUDZINSKI, M.L. Ethology of free-ranging domestic animals. Netherlands: ESPC, 1978. 168p.

BAGSHAW, C.S.; THORROLD, B., DAVISON, M. et al. Cows prefer streams to thougs in all seasons, Florianópolis, SC, 2000. In: INTERNATIONAL CONGRESS OF THE ISAE, 34., 2000, Florianópolis. Anais... Florianópolis: Universidade Federal de Santa Catarina, 2000. 112p.

BAILEY, D.W. et al. Mechanisms that result in large herbivore grazing distribuition patterns. Journal of Range Management, v.49, p.386-400, 1996.

BAUMONT, R.; PRACHE, S.; MEREUT, M. et al. How forage characteristics influence behaviour and intake in small ruminants: a review. Livestock Production Science, v.64. p.15-28, 2000 .

BLACK, J.L; KENNEY, P.A. Factors affecting diet selection by sheep. II. Height and density of pasture. Australian Journal of Agricultural Research, v.35, p.565-578, 1984.

CANGIANO, C.A.; GALLI, J.R.; FERNANDEZ, H.H. Conpast 3.0: Programa de computación para la estimación del consumo de bovinos en pastoreo. Uma aplicación em sistemas lecheros. In: SIMPÓSIO INTERNACIONAL DE PRODUÇÃO ANIMAL. MODELOS PARA A TOMADA DE DECISÕES NA PRODUÇÃO DE BOVINOS E OVINOS, 2., 2002, Santa Maria. Anais... Santa Maria. 2002. p.69-91.

CARVALHO, P.C.F. A estrutura da pastagem e o comportamento ingestivo de ruminantes em pastejo. In: SIMPÓSIO SOBRE AVALIAÇÃO DE PASTAGENS COM ANIMAIS, 1997, Maringá. Anais... Maringá: Universidade Estadual de Maringá, 1997. p.25-52.

CARVALHO, P.C.F; RIBEIRO FILHO, H.M.N; POLI, C.H.E.C. et al. Importância da estrutura da pastagem na ingestão e seleção de dietas pelo animal em pastejo. A produção animal na visão dos brasileiros. Piracicaba: Fundação de Estudos Agrários Luiz de Queiroz, 2001. p.853-871.

CHARNOV, E.L. Optimal foraging, the marginal value theorem. Theoretical Population Biology, v.9, p.129-136, 1976.

DENARDIN-SALDANHA, C.E. Avaliação do rendimento e composição botânica de uma pastagem natural e da dieta 
selecionada por animais em pastejo. Santa Maria: Universidade Federal de Santa Maria, 1989. 159p. Dissertação (Mestrado em Zootecnia) - Universidade Federal de Santa Maria, 1989.

DUMONT, B.; D'HOUR P.; PETIT, M. The usefulness of grazing tests for studying the ability of sheep and cattle to exploiet reproductive patches of pastures. Applied Animal Behaviour Science, v.45, p.79-88, 1995.

DUMONT, B.; DUTRONC, A.; PETIT, M. How readily sheep walk for a preferred forage? Journal of Animal Science, v.76, p.965-971, 1998.

EDWARDS, G.R.; NEWMAN; J.A.; PARSONS A.J. et al. Use of cues by grazing animals to locate food patches: an example with sheep. Applied Animal Behaviour Science, v.51, p.59-68, 1997.

HARVEY, A.; ORR, R.J. Dietary preference of sheep for grass and clover at contrasting sward surface heights. Brazilian Society of Animal Science, Winter Meeting, 1996. 161p.

LACA, E.A.; UNGAR, E.D.; SELIGMAN, N.G.; et al. An integrated methodology for studying short-term grazing behaviour of cattle. Grass and Forage Science, v.47, p.81-90, 1992.

LACA, E.A.; LEMAIRE, G. Measuring sward structure. In: t' MANNETJE, L.; JONES, R.M. (Eds.). Field and laboratory methods for grassland and animal production research. Wallingford: CAB International, 2000. p.103-122.

MORENO, J.A. Clima do Rio Grande do Sul. Porto Alegre: Secretaria da Agricultura, 1961. p.41.

MOTT, G.O.; LUCAS, H.L. The design conduct and interpretation of grazing trials on cultivated and improved pastures. In: INTERNACIONAL GRASSLAND CONGRESS, 6., 1952, Pensylvania. Proceedings... Pensylvania: State College Press, 1952. p.1380-1395.

PILLAR, V.D.P. Multivariate exploratory analysis and randomization testing with MULTIV. Coenoses, v.12, p.145$148,1997$.

PRACHE, S; ROGUET, C.; LOUAULT F. et al. Evolution deschoix alimentaires d'ovins entre talles végétatives et épiées ao cours de l'exploitation d'un couvert épié de dactyle. Rencontres Recherches Ruminants, v.3, p.89-92, 1996.

PRACHE, S.; ROGUET, C.; PETIT, M. How degree of selectivity modifies foraging behaviour of dry ewes on reproductive compared to vegetative sward structure. Applied Animal Behaviour Science, v.57, p.91-108, 1998.

QUADROS, F.L.F.; TREVISAN, N.B.; SILVA, A.C.F. et al. Preferência por sítios de pastejo em pastagem de aveia e azevém submetida a diferentes biomassas de lâmina foliar verde. In.: REUNIÃO ANUAL DA SOCIEDADE BRASILEIRA DE ZOOTECNIA, 40., 2003, Santa Maria. Anais... Santa Maria: Sociedade Brasileira de Zootecnia, 2003. (CD-ROM)
ROUGE, C.; PRACHE, S.; PETIT, M. Feeding station behaviour of ewes in response to forage availability and sward phonological stage. Applied Animal Behaviour Science, v.56, 187-201, 1998.

SILVA, A.C.F.; QUADROS, F.L.F; BANDINELLI, D.G. et al. (a) Produção animal em pastagem de aveia preta mais azevém sob diferentes níveis de biomassa de lâmina foliar verde. In: REUNIÃO ANUAL DA SOCIEDADE BRASILEIRA DE ZOOTECNIA, 40., 2003, Santa Maria. Anais... Santa Maria: Sociedade Brasileira de Zootecnia, 2003. (CD-ROM)

SILVA, A.C.F.; QUADROS, F.L.F.; TREVISAN, N.B. et al. (b) Comportamento ingestivo e taxa de bocados de terneiros de corte em pastagem de estação fria sob diferentes níveis de biomassa de lâmina foliares verdes. In: REUNIÃO ANUAL DA SOCIEDADE BRASILEIRA DE ZOOTECNIA, 40., 2003. Santa Maria, Anais... Santa Maria: Sociedade Brasileira de Zootecnia, 2003. (CD-ROM)

SOARES, A.B. Efeito da alteração da oferta de matéria seca de uma pastagem natural sobre a produção animal e dinâmica da vegetação. Porto Alegre: Universidade Federal do Rio Grande do Sul, 2001. 180p. Tese (Doutorado em Plantas Forrageiras) - Universidade Federal do Rio Grande do Sul, 2001.

STEPHEN, D.W.; KREBS, J.R. Foraging theory. Princeton: Princeton University Press, 1986. 247p.

t' MANNETJE, L. Measuring biomass of grassland vegetation. In: t'MANNETJE, L.; JONES, R.M. (Eds.) Field and laboratory methods for grassland and animal production research. Cambridge: CABI, 2000. p.151-178.

TREVISAN, N.B.; QUADROS, F.L.F.; SILVA, A.C.F. et al. Tempo de permanência por estação alimentar e distância entre estações de pastejo em pastagem de aveia preta e azevém submetida a diferentes níveis de biomassa de lâmina foliar verde. In: REUNIÃO ANUAL DA SOCIEDADE BRASILEIRA DE ZOOTECNIA, 40., 2003, Santa Maria, Anais... Santa Maria: Sociedade Brasileira de Zootecnia, 2003. (CD-ROM)
Recebido em: 04/03/04 Aceito em: 07/03/05 